\title{
Pediatric multicompartmental trigeminal schwannoma: illustrative case
}

\author{
Alexander P. Landry, MD, ${ }^{1}$ Vincent C. Ye, MD, ${ }^{1}$ Kerry A. Vaughan, MD, ${ }^{1,2}$ James M. Drake, MBBCh, MSc, ${ }^{1,2}$ Peter B. Dirks, MD, PhD, ${ }^{1,2}$ \\ and Michael D. Cusimano, MD, $\mathrm{PhD}^{1-3}$
}

${ }^{1}$ Divison of Neurosurgery, University of Toronto, Toronto, Ontario, Canada; ${ }^{2}$ Division of Neurosurgery, Hospital for Sick Children, Toronto, Ontario, Canada; and ${ }^{3}$ Division of Neurosurgery, St. Michael's Hospital, Toronto, Ontario, Canada

\begin{abstract}
BACKGROUND Trigeminal schwannoma (TS) is an uncommon and histologically benign intracranial lesion that can involve any segment of the fifth cranial nerve. Given its often impressive size at diagnosis and frequent involvement of critical neurovascular structures of the skull base, it represents a challenging entity to treat. Pediatric TS is particularly rare and presents unique challenges. Similarly, tumors with extension into multiple compartments (e.g., middle cranial fossa, posterior cranial fossa, extracranial spaces) are notoriously difficult to treat surgically. Combined or staged surgical approaches are typically required to address them, with radiosurgical treatment as an adjunct.
\end{abstract}

OBSERVATIONS The authors presented the unusual case of a 9-year-old boy with a large, recurrent multicompartmental TS involving Meckel's cave, the cerebellopontine angle, and the infratemporal fossa. Near-total resection was achieved using a frontotemporal-orbitozygomatic craniotomy with a combined interdural and extradural approach.

LESSONS The case report adds to the current literature on multicompartmental TSs in children and their management. The authors also provided a simplified classification of TS that can be generalized to other skull base tumors. Given a lack of precedent, the authors intended to add to the discussion regarding surgical management of these rare and challenging skull base lesions.

https://thejns.org/doi/abs/10.3171/CASE2171

KEYWORDS trigeminal schwannoma; pediatric; skull base; anatomical classification; surgical approaches

Trigeminal schwannoma (TS) is a rare entity, representing less than $0.5 \%$ of intracranial neoplasms and approximately $0.8 \%$ to $8 \%$ of intracranial schwannomas overall. ${ }^{1,2}$ Peak incidence occurs in the third and fourth decades of life; pediatric cases are exceedingly rare, with only a handful having been reported in the literature. ${ }^{3-7}$ Patients may present with trigeminal nerve dysfunction, headaches, dysphagia, or diplopia (although many cases are discovered incidentally). ${ }^{4}$ These tumors exhibit considerable anatomical heterogeneity and can originate from or involve any segment of the trigeminal nerve. Consequently, they can be found in anatomical permutations of the posterior cranial fossa; middle cranial fossa/Meckel's cave; and, rarely, extracranial spaces (e.g., orbit, pterygopalatine fossa, or infratemporal fossa). ${ }^{8}$ Importantly, tumors with tricompartmental extension (i.e., extracranial, middle fossa, and posterior fossa) are particularly rare and challenging to treat.

Management of multicompartmental TS in general is challenging and demands individualized multidisciplinary input. ${ }^{9}$ Treatment goals include improvement in neurological symptoms, preservation of cranial nerve function, and prevention of recurrence/progression. Stable lesions may be monitored with serial imaging, and stereotactic radiosurgery has shown some promise in improving progression-free survival. ${ }^{10}$ Resection remains the gold standard treatment, but the choice of approach is nuanced and depends largely on tumor anatomy and surgeon comfort. For both adult and pediatric multicompartmental lesions, surgical corridors may include combinations of presigmoid, frontotemporal, subtemporal/ transpetrosal, and two-stage middle and posterior fossa exposures, ${ }^{2-6,11}$ with or without the addition of endonasal endoscopic ${ }^{2,12-16}$ or transfacial (transmaxillary/transmandibular) approaches to target significant infratemporal extension. ${ }^{4,11,17-19}$

Management of TS in pediatric patients presents additional challenges because of anatomical differences, the potential for impact on development, and the need to minimize blood loss. Balancing treatment goals, including curative complete resection and minimizing

ABBREVIATIONS MPE = middle fossa/posterior fossa/extracranial; MRI = magnetic resonance imaging; TS $=$ trigeminal schwannoma. INCLUDE WHEN CITING Published online May 17, 2021; DOI: 10.3171/CASE2171.

SUBMITTED February 5, 2021. ACCEPTED February 28, 2021.

(c) 2021 The authors, CC BY-NC-ND 4.0 (http://creativecommons.org/licenses/by-nc-nd/4.0/). 
morbidity, should occur in the context of the longer relative life expectancy of a child compared to that of an adult patient. The postoperative morbidity of extensive, combined, or two-stage approaches may be well tolerated in terms of acute recovery, for example, but may drastically impact development of a child. Alternatively, the long-term morbidity associated with the wide-field radiation reserved for residual or recurrent lesions of all types in children is also well known and cannot be overemphasized. ${ }^{20,21}$

Multicompartmental TSs with significant infratemporal extension are particularly difficult lesions to treat surgically and are rare in children. In fact, we are unable to identify a previously reported case in the literature. In this study, we highlight a challenging case of a recurrent pediatric TS with significant trifossa extension resected using a single frontotemporalorbitozygomatic exposure, with access of the tumor through interdural and extradural corridors.

\section{Illustrative Case}

We present the case of a 9-year-old boy with a complex history of recurrent TS. He was initially diagnosed at 4 years of age when computed tomography and subsequent magnetic resonance imaging (MRI) for unrelated periorbital swelling revealed a large contrast-enhancing lesion centered in the right masticator space and extending through the foramen ovale along the path of the mandibular trigeminal division (Fig. 1A). He underwent biopsy in 2014 that revealed TS (World Health Organization grade I) and subsequent subtotal resection via frontotemporal-orbitozygomatic craniotomy in 2015 . The tumor was particularly fibrous, and access to the caudal pole of the infratemporal portion of the tumor could not be safely resected during this initial approach because of cardiac instability, possibly as a result of manipulation (Fig. 1B). The patient underwent follow-up serial imaging and demonstrated
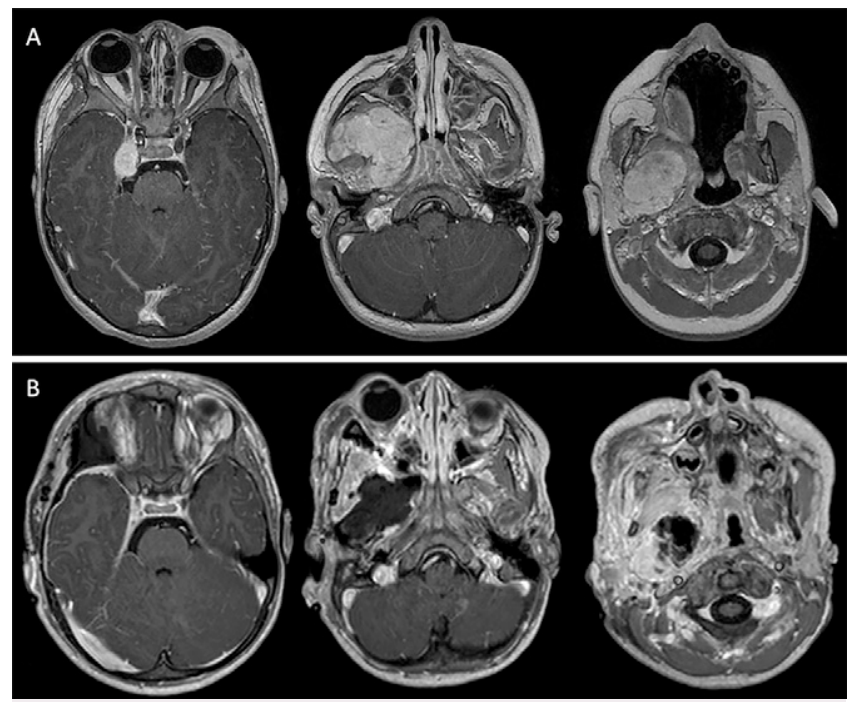

FIG. 1. Initial preoperative (2014) and postoperative (2015) MRI. A: T1weighted axial MRI with gadolinium enhancement reveals a large, homogeneously enhancing, dumbbell-shaped lesion involving the masticator space, Meckel's cave, the cerebellopontine angle, and the infratemporal fossa. Communication between intracranial and extracranial components is via a remodeled, expanded foramen ovale. B: Postoperative MRI reveals subtotal resection of the lesion, with residual tumor in the infratemporal fossa. progressive recurrence of both the infratemporal and middle fossa components over time, beginning in 2016 and with the most significant growth shown on MRI in October 2018. Notably, invasion into the cavernous sinus was difficult to assess preoperatively, although the tumor did not encase the internal carotid artery and remained entirely medial to an extended styloid process. He underwent transcervical debulking by our otolaryngology colleagues in March 2019, which confirmed that the tumor pathology was unchanged. After extensive multidisciplinary discussions, repeat frontotemporal-orbitozygomatic craniotomy was performed in October 2020 (Fig. 2) with a second-stage transmandibular approach to address any potential infratemporal residual if needed.

Preoperatively, the patient reported 6 months of intermittent headaches, jaw pain, right-sided facial pain, and a globus sensation with increasing fullness of the parapharyngeal region. There was no objective facial numbness or weakness, nor was there asymmetry in the muscles of mastication. The remainder of his neurological exam was unremarkable. The patient will be screened for neurofibromatosis, but there were no other clinical manifestations or positive family history.

Intraoperatively, the patient was positioned supine with standard $45^{\circ}$ head turn; neurophysiological monitoring included brainstem auditory evoked potentials, cortical and brainstem/cervical sensory evoked potentials, motor evoked potentials, and electromyography monitoring of cranial nerves III-VII and IX-XII. A two-piece orbitozygomatic craniotomy was extended to unroof the anterior half of the remodeled foramen ovale. The middle fossa and the infratemporal fossa were connected by removing the middle fossa floor, thus reducing the access to two cavities. The tumor component within Meckel's cave was accessed interdurally by dissecting between the dural layers starting at the orbitomeningeal band and superior orbital fissure, incising and reflecting only the periosteal dural layer posteriorly and so connecting the compartment of the tumor in Meckel's cave to the infratemporal compartment. The tumor was
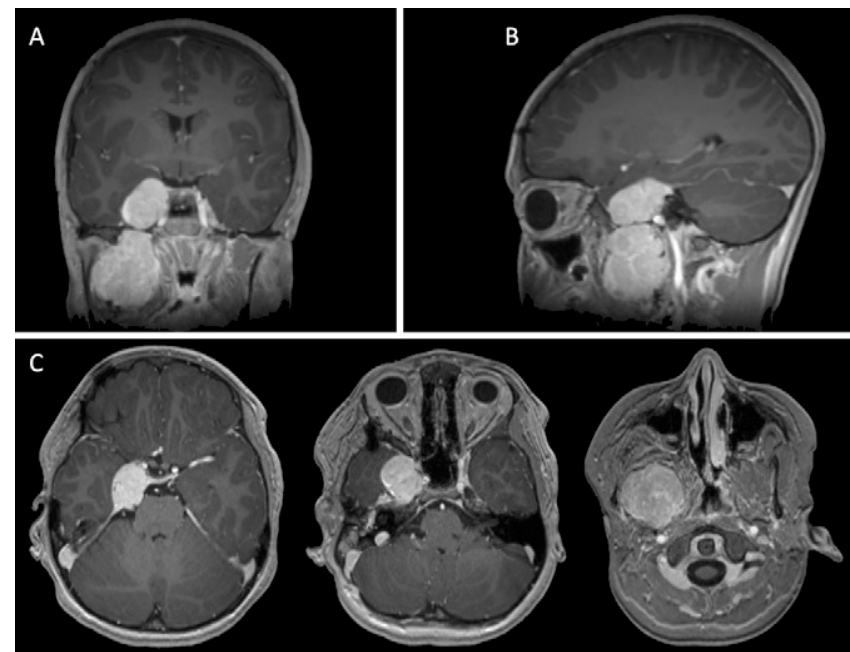

FIG. 2. Preoperative MRI (2020). T1-weighted MRI with gadolinium enhancement in coronal (A), sagittal (B), and axial (C) planes reveals significant regrowth of the schwannoma. As it had previously, the lesion involves Meckel's cave and extends inferiorly into the infratemporal fossa and medially into the cerebellopontine angle, with compression of the cavernous sinus. 
subsequently resected until viable mandibular nerve fibers limited its removal posteriorly (Fig. 3).

Access to the small extension of tumor into the posterior fossa was facilitated by its erosion of the anterior petrous apex; following it superiorly and posteriorly to the petrous apex allowed for complete removal of this component. Importantly, this access to the cerebellopontine angle was actually subdural; thus, our approach did not require intradural access. At this point, we had traced out the whole three-dimensional resection volume by connecting all three compartments. The infratemporal extension was then aggressively debulked through the previously exposed foramen ovale until no more tumor could be pulled into the field of view. This was facilitated by neuronavigation and the presence of a particularly large styloid process of bone, which provided confirmation of surgical landmarks. Neurophysiological monitoring remained at preoperative baseline throughout the case. Because this was an entirely interdural and extradural approach, no significant reconstruction was required beyond the usual hemostatic agents and sealants.

The patient recovered well postoperatively, with mild new right facial V2 and V3 hypesthesia and a transient mild right-sided facial weakness. Given the recent surgery, we continue to await longer term follow-up. Postoperative MRI revealed near-total resection of the tumor, with a thin residual along the margin of resection in the infratemporal fossa (Fig. 4). Final pathology remained consistent with schwannoma, with an estimated Mib-1 proliferation index of $3 \%$ to $4 \%$. Given the near-total resection of the tumor via the extended orbitozygomatic craniotomy, the staged transmandibular approach was canceled. In light of the recurrent nature of the tumor
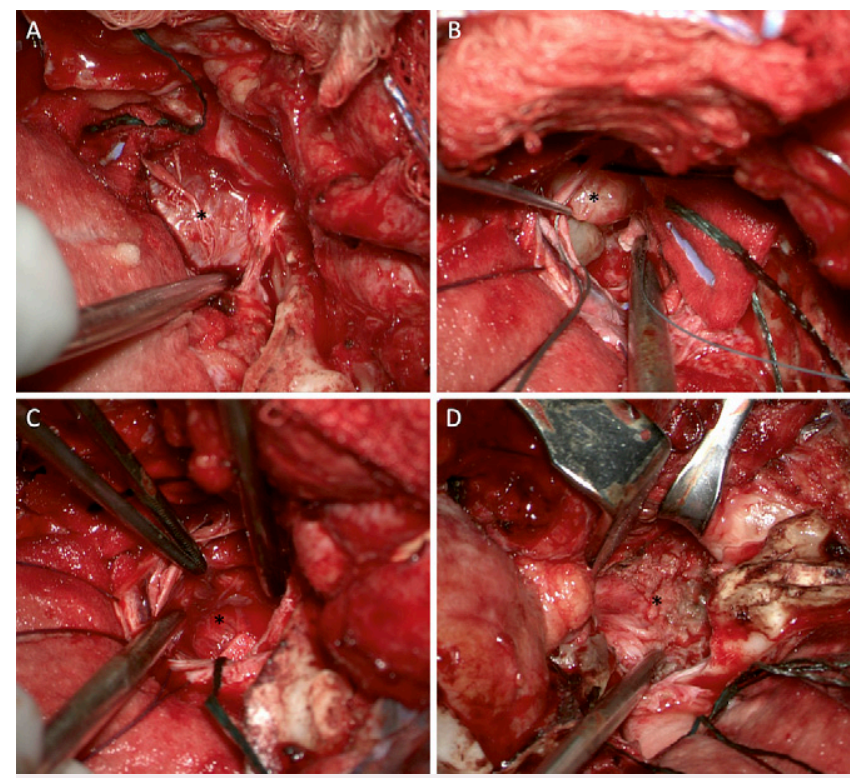

FIG. 3. Intraoperative dissection. A: Extradural exposure of middle fossa portion of the tumor, which was found to be compressing but not invading the cavernous sinus. Retraction toward the ipsilateral globe in the bottom left of the image. B: Intradural tumor resection from within Meckel's cave. C: Resection cavity in Meckel's cave, with nerve fibers draped over a small residual posteriorly. D: Exposure of the lesion's infratemporal extension through an expanded foramen ovale. Asterisks indicate tumor. and small residual, the multidisciplinary consensus plan for followup included adjuvant outpatient radiotherapy once the tumor cavity involutes.

\section{Discussion}

\section{Observations}

Multicompartmental TS is a rare and challenging neurosurgical disease; to our knowledge, it has yet to be reported in the pediatric population. Achieving satisfactory resection is critical for optimizing outcome, but care must be taken to avoid undue surgical morbidity. Such morbidity can be magnified across a lifetime in pediatric cases given the ongoing neurodevelopment during childhood, particularly because of the role of the trigeminal nerve in craniofacial development. In cases with infratemporal extension, transfacial exposures have often been used in adult patients in addition to traditional skull base osteotomies. We present a rare case of a recurrent multicompartmental TS in a child who underwent successful resection of significant infratemporal extension via a wide orbitozygomatic approach, which obviated the need for a staged transfacial approach without adding significant surgical morbidity. Given the unusually aggressive nature of his tumor and small residual, localized radiation was offered despite its potential side effects. However, it was believed that the extensive resection achieved in this case would allow for significantly less cerebral irradiation. Other surgical options for this case include extended endonasal approaches or other skull base exposures (i.e., presigmoid, subtemporal), possibly combined with a staged transmandibular approach. However, the extremely fibrous nature of the tumor would have been a major limitation for endoscopic approaches. In our opinion, the extended orbitozygomatic approach was able to minimize morbidity while maximizing resection.

Although the selection of a surgical approach in adult skull base surgery can be nuanced in difficult cases, pediatric cases provide their own unique challenges mainly because of anatomical differences and the rarity of pediatric skull base pathology. Development
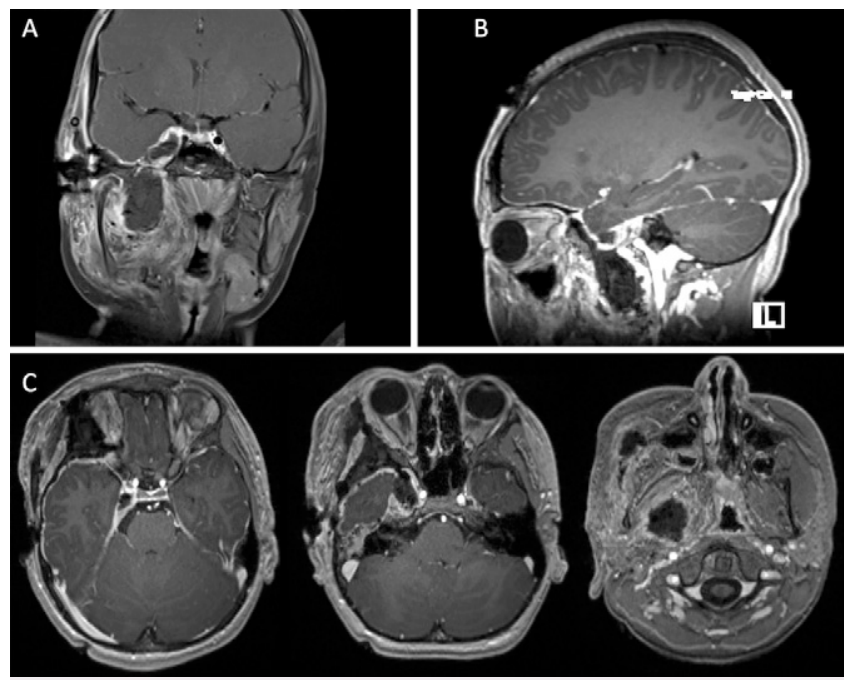

FIG. 4. Postoperative MRI (2020). T1-weighted MRI with gadolinium enhancement in coronal (A), sagittal (B), and axial (C) planes shows near-total resection of the lesion. There is a thin peripheral residual in the infratemporal fossa. 
of the skull base is complex and beyond the scope of this study, although it should be noted that there is fusion of the anterior and posterior synchondroses by age 8 years, with attainment of nearly adult proportions by age $10 .^{22,23}$ Although most traditional skull base approaches can be transferred to the pediatric population of all ages, age-dependent considerations are needed. In an orbitozygomatic craniotomy, one must be mindful of the following factors: the zygoma is poorly developed in young children, and its osteotomy may be associated with changes in facial growth patterns; the supraorbital foramen/notch is often absent in children younger than 8 ; and the pterion is displaced anteriorly in children. ${ }^{23}$ For lateral skull base (transpetrous) approaches, considerations include the possible lack of mastoid pneumatization in children and a lower rate of cerebrospinal fluid leakage. ${ }^{24}$

One study found that the degree of sphenoid pneumatization did not affect outcome in children who underwent endoscopic endonasal resection of craniopharyngioma, ${ }^{25}$ although it remains an important age-related consideration and may be a critical factor in approach selection. More generally, hemostasis is of particular importance in children because they have smaller circulating blood volume, and relatively immature prominent bony facial features may affect the accuracy of neuronavigation. ${ }^{23,24,26}$ Finally, it must not be overlooked that pediatric skull base tumors represent only approximately $5 \%$ of skull base tumors overall (with differing pathology), and their surgical management has received little attention in the neurosurgical literature. ${ }^{23,26}$ The rarity of pediatric skull base surgery necessitates the involvement of a multidisciplinary team, meticulous preoperative planning, and often collaboration between an adult skull base neurosurgeon and a pediatric neurosurgeon.

The role of the trigeminal nerve in development must not be forgotten during preoperative planning for children with trigeminal lesions. Although the mechanism remains poorly understood, multiple cranial nerves have been shown to exert an important influence on craniofacial development; specifically, lesion studies have demonstrated the critical role of the trigeminal nerve in odontogenesis. ${ }^{27}$ Additionally, muscle atrophy secondary to trigeminal dysfunction can lead to significant facial asymmetry, ${ }^{28}$ which may have developmental consequences in a child. Therefore, preservation of trigeminal nerve function is an important preoperative consideration. To this end, consideration of lesion etiology is critical to surgical planning and decision-making. Schwannomas arise from the nerve sheath and therefore compress, rather than infiltrate, the nerve itself. It is possible to resect these tumors without sustaining damage to the nerve, although even with modern microsurgical techniques, it is rare to achieve complete resection without at least transient postoperative nerve dysfunction. ${ }^{29}$

By contrast, one must consider the differential diagnosis of neurofibroma, particularly in children with neurofibromatosis type 1 , which originates from the nerve or perineural cells. ${ }^{30}$ This diagnosis necessitates destruction of the nerve to achieve resection, with potentially significant consequences for development. In general, neurofibroma resection should be limited to the offending component rather than extirpation of all the involved branches of the trigeminal nerve, which would not be feasible or advisable. Additionally, schwannomas involve only one division of the trigeminal nerve, whereas neurofibromas generally involve all divisions and subsequent branches. Therefore, we encourage careful review of preoperative imaging with this in mind. With similar motivation, we also
TABLE 1. Proposed anatomical classification of TS

\begin{tabular}{lcc}
\hline Type & Compartments & Classification \\
\hline Single fossa "X" & Middle fossa & $\mathrm{A}$ \\
\hline & Posterior fossa & $\mathrm{B}$ \\
\hline & Extracranial & $\mathrm{C}$ \\
\hline Dual fossa "AX" & Middle fossa \& posterior fossa & $\mathrm{AB}$ \\
\hline & Middle fossa \& extracranial & $\mathrm{AC}$ \\
\hline Trifossa & $\begin{array}{c}\text { Extracranial \& middle fossa } \\
\text { \& posterior fossa }\end{array}$ & $\mathrm{ABC}$ \\
\hline
\end{tabular}

advocate the routine use of intraoperative frozen sections and intraoperative neuromonitoring.

We conclude our discussion of complex presentations of TS by proposing an update to previous classification systems, of which there are many. ${ }^{31,32}$ In particular, the middle fossa/posterior fossa/extracranial (MPE) classification is a commonly used system that helps compartmentalize tumors by anatomical modules to systematize surgical decision-making. We propose a simplified version of this wellestablished paradigm: the $A B C$ classification (Table 1). In this system, single-compartment tumors are represented by $X$, dual fossa tumors by $A X$, and trifossa tumors by $A B C(A=$ middle fossa, $B=$ posterior fossa, $C=$ extracranial, $X=$ any compartment). Like the MPE classification, our classification yields clear subgroups with a defined set of surgical options. The advantage of the proposed system, however, lies in its simplification of the notion that any multicompartmental lesion must involve the middle fossa and its reductionist formulation, such that tumors are dichotomized into single compartment $(A / B / C)$ versus multicompartment $(A+B / C / B C)$ in a particularly simple way. This system, in turn, allows for an increasingly systematic approach to even the most complex lesions.

\section{Lessons}

Multicompartmental TS with infratemporal extension is a notoriously challenging surgical pathology, with many viable operative approaches. There is particular challenge in the pediatric population because of the lesion's extreme rarity, anatomical and developmental considerations, and lack of surgical precedent. We present the case of a large, recurrent multicompartmental TS in a child who underwent near-total resection via frontotemporal-orbitozygomatic craniotomy alone. Additionally, we propose a simplification of current classification models of TS. We hope this case provides insight into management options for these rare lesions and the role of single-stage transcranial exposures in select cases.

\section{References}

1. McCormick PC, Bello JA, Post KD. Trigeminal schwannoma. Surgical series of 14 cases with review of the literature. J Neurosurg. 1988;69(6):850-860.

2. Li M, Wang $X$, Chen $G$, et al. Trigeminal schwannoma: a singlecenter experience with 43 cases and review of literature. $\mathrm{Br} \mathrm{J} \mathrm{Neu-}$ rosurg. 2021;35(1):49-56.

3. Verstappen CCP, Beems T, Erasmus CE, van Lindert EJ. Dumbbell trigeminal schwannoma in a child: complete removal by a one-stage pterional surgical approach. Childs Nerv Syst. 2005;21(11):1008-1011.

4. Ramina R, Mattei TA, Sória MG, et al. Surgical management of trigeminal schwannomas. Neurosurg Focus. 2008;25(6):E6. 
5. O'Connor KP, Pelargos PE, Palejwala AH, et al. Resection of pediatric trigeminal schwannoma using minimally invasive approach: case report, literature review, and operative video. World Neurosurg. 2019;127:518-524.

6. Bertot BE, Presti ML, Stormes K, et al. Trigeminal schwannoma presenting with malocclusion: a case report and review of the literature. Surg Neurol Int. 2020;11:230.

7. Holman MA, Schmitt WR, Carlson ML, et al. Pediatric cerebellopontine angle and internal auditory canal tumors: clinical article. J Neurosurg Pediatr. 2013;12(4):317-324.

8. Chung JC, Chung SY, Kim SM, Park MS. Surgery for a case of three-compartment trigeminal schwannoma: technical aspects. J Korean Neurosurg Soc. 2010;48(4):383-387.

9. Makarenko S, Ye V, Akagami R. Natural history, multimodal management, and quality of life outcomes of trigeminal schwannomas. $J$ Neurol Surg B Skull Base. 2018;79(6):586-592.

10. Kano H, Niranjan A, Kondziolka D, et al. Stereotactic radiosurgery for trigeminal schwannoma: tumor control and functional preservation. Clinical article. J Neurosurg. 2009;110(3):553-558.

11. Mahajan A, Sable NP, Vaish R, et al. Multicompartmental trigeminal schwannomas: dumbbell tumors revisited. J Glob Oncol. 2016;2(6): 431-435.

12. Jacquesson $T$, Berhouma M, Picart T. Total removal of a trigeminal schwannoma via the expanded endoscopic endonasal approach. Technical note. Acta Neurochir (Wien). 2015;157:935-938.

13. Almomen A, Alyousif A, Ali Z, et al. Image-guided endonasal endoscopic excision of Meckel's cave trigeminal schwannoma from cavernous and petrous carotid artery. J Surg Case Rep. 2020;9:1-5.

14. Alyousif A, Almolani F, Almomen A. Endonasal endoscopic removal of a large intracranial infratemporal fossa trigeminal schwannoma: a case report and literature review. Otolaryngol Case Reports. 2020; 17:100220.

15. Raza SM, Donaldson AM, Mehta A, et al. Surgical management of trigeminal schwannomas: defining the role for endoscopic endonasal approaches. Neurosurg Focus. 2014;37(4):E17.

16. Shi J, Chen J, Chen T, et al. Neuroendoscopic resection of trigeminal schwannoma in the pterygopalatine/infratemporal fossa via the transnasal perpendicular plate palatine bone or transnasal maxillary sinus approach. World Neurosurg. 2018;120:e1011-e1016.

17. Kouyialis AT, Stranjalis G, Papadogiorgakis N, et al. Giant dumbbell-shaped middle cranial fossa trigeminal schwannoma with extension to the infratemporal and posterior fossae. Acta Neurochir (Wien). 2007;149(9):959-964.

18. Alafaci C, Caffo M, Barresi V, et al. Large trigeminal schwannoma of the infratemporal fossa: evaluation of neoangiogenesis in this rare neoplasm. Head Neck. 2013;35(9):E272-E276.

19. Politi M, Toro C, Sbuelz M. A giant trigeminal schwannoma of the infratemporal fossa removed by transmandibular approach and coronoidectomy. Oral Maxillofac Surg Cases. 2016;2(1):10-13.

20. Tsang DS, Kim L, Liu ZA, et al. Intellectual changes after radiation for children with brain tumors: which brain structures are most important? Neuro Oncol. 2021;23(3):487-497.

21. Cantelmi D, Schweizer TA, Cusimano MD. Role of the cerebellum in the neurocognitive sequelae of treatment of tumours of the posterior fossa: an update. Lancet Oncol. 2008;9(6):569-576.
22. Gruber DP, Brockmeyer D. Pediatric skull base surgery. 1. Embryology and developmental anatomy. Pediatr Neurosurg. 2003;38(1):2-8.

23. LoPresti MA, Sellin JN, DeMonte F. Developmental considerations in pediatric skull base surgery. J Neurol Surg B Skull Base. 2018; 79(1):3-12.

24. Kazahaya K. Lateral skull base approaches in pediatric skull base surgery. J Neurol Surg B Skull Base. 2018;79(1):47-57.

25. Kuan EC, Kaufman AC, Lerner D, et al. Lack of sphenoid pneumatization does not affect endoscopic endonasal pediatric skull base surgery outcomes. Laryngoscope. 2019;129(4):832-836.

26. Brockmeyer D, Gruber DP, Haller J, et al. Pediatric skull base surgery. 2. Experience and outcomes in 55 patients. Pediatr Neurosurg. 2003;38(1):9-15.

27. Sudiwala S, Knox SM. The emerging role of cranial nerves in shaping craniofacial development. Genesis. 2019;57(1):e23282.

28. Braun JS, Hahn K, Bauknecht HC, Schielke E. Progressive facial asymmetry due to trigeminal motor neuropathy. Eur Neurol. 2006; 55(2):96-98.

29. Chen LF, Yang Y, Yu XG, et al. Operative management of trigeminal neuromas: an analysis of a surgical experience with 55 cases. Acta Neurochir (Wien). 2014;156(6):1105-1114.

30. Inoue T, Elaskary M, Shima A, et al. Trigeminal neurofibroma in the infratemporal fossa arising from the inferior alveolar nerve: a case report. Mol Clin Oncol. 2017;7(5):825-829.

31. Niranjan A, Barnett S, Anand V, Agazzi S. Multimodality management of trigeminal schwannomas. J Neurol Surg B Skull Base. 2016;77(4):371-378.

32. Yoshida K, Kawase T. Trigeminal neurinomas extending into multiple fossae: surgical methods and review of the literature. J Neurosurg. 1999;91(2):202-211.

\section{Disclosures}

The authors report no conflict of interest concerning the materials or methods used in this study or the findings specified in this paper.

\section{Author Contributions}

Conception and design: Landry, Ye, Vaughan, Drake, Cusimano. Acquisition of data: Landry, Ye, Vaughan, Cusimano. Analysis and interpretation of data: Landry, Ye, Cusimano. Drafting the article: Landry, Ye, Drake. Critically revising the article: Landry, Ye, Vaughan, Drake, Cusimano. Reviewed submitted version of manuscript: Landry, Ye, Vaughan, Cusimano. Approved the final version of the manuscript on behalf of all authors: Landry. Statistical analysis: Ye. Administrative/ technical/material support: Dirks. Study supervision: Cusimano.

\section{Correspondence}

Alexander P. Landry: University of Toronto, Toronto, ON, Canada. alex.landry@mail.utoronto.ca. 